\title{
Localized Adhesion of Nongerminated Venturia inaequalis Conidia to Leaves and Artificial Surfaces
}

\author{
C. F. A. Schumacher, U. Steiner, H.-W. Dehne, and E.-C. Oerke
}

Institute of Crop Science and Resource Conservation, Phytomedicine, University of Bonn, Nussallee 9, D-53115 Bonn, Germany. Accepted for publication 16 March 2008.

\begin{abstract}
Schumacher, C. F. A., Steiner, U., Dehne, H.-W., and Oerke, E.-C. 2008. Localized adhesion of nongerminated Venturia inaequalis conidia to leaves and artificial surfaces. Phytopathology 98:760-768.

Adhesion to the host surface is the first step for successful plant pathogen development and has been reported to be associated with both passive and active processes. For conidia of Venturia inaequalis, which depend on leaf wetness for germination, this process has not yet been described. Conidia of $V$. inaequalis adhered to wet hydrophobic surfaces immediately after contact to the surface, hours before initiation of germination. Attachment of nongerminated conidia was much better on hydrophobic surfaces, such as apple leaves and polystyrene, than on hydrophilic glass. Conidia released adhesive material localized in a drop-

contact surface only when water was present. Histochemical investigations indicated the presence of proteins and carbohydrates in STG, lectin labeling the presence of $\beta$-galactose and $\mathrm{N}$-acetylglucosaminyl residues. Transmission electron microscopy revealed two phases in the STG at the tip of dry mature conidia; as STG was present on the outer side of the intact fungal cell wall its formation should be associated with the secretion of glue through pores of the conidial wall. Surface-active substances affected the adhesion of conidia to hydrophobic surfaces stressing the importance of hydrophobic interactions. The use of protein biosynthesis inhibitors did not affect adhesion of conidia indicating that the adhesive material was preformed. It is concluded that the coincidence of STG, contact to a hydrophobic surface, and free water are essential for the adhesion of $V$. inaequalis conidia.
\end{abstract} let named spore tip glue (STG) at the spore apex which interacted with a
Adhesion has been shown to be a key step in the establishment of pathogens in animals and plants $(19,36)$. Firm attachment of pathogen cells to the surface of a potential host not only anchors them in a favorable position for host cell penetration but also is often a prerequisite for the development of specialized infection structures required for penetration $(7,11,16,32)$. Without adhesion, conidia can be displaced more easily from the infection court by factors such as rainfall, wind, and competition from other pathogens (36).

Scab caused by the ascomycete Venturia inaequalis (Cooke) G. Winter is the most important disease of apple worldwide (22). For germination of ascospores in spring, leaf wetness over a period of 10 to $20 \mathrm{~h}$ linked to temperatures ranging between 10 and $20^{\circ} \mathrm{C}$ is required (23). After infection and establishment in the host tissue, conidia are produced which are distributed by rain and splashes of water to infect other leaves during the summer. Conidia are only able to germinate in the presence of free water (22). Hence, nongerminated conidia of $V$. inaequalis must be able to adhere to wet surfaces. For many fungal pathogens adhesion is known to be a process of secretion of adhesive materials prior to germination $(14,27)$. Most fungal adhesives appear to be glycoproteins (21, $25,40)$. Although the general mechanism of spore attachment is common among pathogenic fungi (4), there is variation between fungal species in the composition of adhesive material, the environmental cues which induce the development of adhesiveness, and the stages of fungal development at which adhesion occurs (11). The attachment of powdery mildew fungi on leaves differs markedly from that of Magnaporthe grisea, largely because free water is not required for prepenetration development (36). Spores like those of $M$. grisea, which usually germinate in water films,

Corresponding author: E.-C. Oerke; E-mail address: ec-oerke@uni-bonn.de

doi:10.1094/PHYTO-98-7-0760

(c) 2008 The American Phytopathological Society are able to develop on wet hydrophobic surfaces without being washed from the leaf. A prominent deposit of adhesive material, called spore tip mucilage (STM) by Hamer et al. (14), is stored in the periplasmic space at the apex of nonhydrated dormant M. grisea conidia. When conidia are hydrated by moist air, rain, or dew, the spore wall at the tip is ruptured and STM is immediately released $(4,14)$.

Adhesion of $M$. grisea is described to be a passive process in which a preformed adhesive is released that allows a rapid attachment to the host surface without expenditure of metabolic energy (14). Also the hydrophobicity of surfaces has an effect on spore adhesion. Conidia of $M$. grisea and Colletotrichum graminicola were found to adhere more tightly to Teflon or polystyrene than to the hydrophilic glass surface $(14,26)$. In contrast, nongerminated conidia of Blumeria graminis adhered more strongly to glass than to hydrophobic barley leaves, because of continued accumulation of conidial extracellular material (ECM) on glass indicating the possibility that de novo synthesis and release contributes to additional active adhesion (39). Attachment of B. graminis is accompanied by release of cutinase from the spore. This enzyme appears not only to fix the spore to the leaf surface but also makes the surfaces of both the attachment zone on the plant surface and the spore more hydrophilic (36). Nicholson and Epstein (27) describe that attachment involves an active process of secretion of adhesive material by a fungus. This process is highly specific for the recognition of and binding to a particular host species. Adhesion in C. graminicola is described as requiring spore metabolism because spores do not achieve maximum adhesion until 30 min after landing on a surface. Mercure et al. (26) suggested that de novo glycoprotein synthesis is involved in the adhesion of nongerminated conidia of $C$. graminicola. Similarly, Nectria haematococca requires respiration and protein synthesis before spores become adhesive (17). Cochliobolus heterostrophus shows a nonspecific attachment to several surfaces. Adhesion results from the release of ECM at the conidial tips prior to emergence of 
the germ tube; however, adhesion begins only about 20 min after hydration (5). A two-layered sheath of ECM was visible at the tips of conidia just prior to germination and also later around the germ tube (12). Deising et al. (9) showed that uredospores of Uromyces viciae-fabae form a film of matrix material between spore and leaf surface when in contact with aqueous media. They isolated two esterases and a cutinase from the surface of uredospores and suggested that firm adhesion of the pads to the leaf surface depends on enzyme activities.

In contrast to a large amount of literature on pre- and postinfectional development of $V$. inaequalis, little is known about the mode of conidial attachment to the host surface. This study focuses on the essential process of conidial adhesion of $V$. inaequalis to different surfaces under wet conditions. We investigated the adhesion of $V$. inaequalis on its natural host and on model surfaces, especially whether adhesion of $V$. inaequalis conidia depends on preformed adhesive material or is an active process involving the secretion of such material after recognition of the surface. The ultrastructure of conidia was investigated in electron microscopic studies; light microscopy was used in time course studies and for characterizing the chemical components of the adhesive.

\section{MATERIALS AND METHODS}

Pathogen. Conidia of $V$. inaequalis strain HS1 from the Institute of Crop Science and Resource Conservation were brushed off infected apple leaves. Dry conidia were stored in 1.5-ml tubes at $4^{\circ} \mathrm{C}$ and were suspended in distilled water to prepare spore suspensions (typically $5 \times 10^{4}$ conidia $\mathrm{ml}^{-1}$ ).

Plant cultivation. Seeds of Malus domestica, cv. Golden Delicious, were treated with $0.1 \%$ Euparen 50 WG (tolylfluanid, Bayer CropScience, Monheim, Germany) for $5 \mathrm{~min}$ and then stored at $4^{\circ} \mathrm{C}$ in the dark for 2 weeks. After sowing in substrate for salt-sensitive plants ( $\mathrm{pH} 5$ to 6 , salt content: $0.8 \mathrm{~g} \mathrm{liter}^{-1}$, Klasmann-Deilmann $\mathrm{GmbH}$, Geeste, Germany), they grew at 18 to $20^{\circ} \mathrm{C}$ and $18 \mathrm{~h}$ daylight in a greenhouse. After 2 weeks individual seedlings were grown in plastic pots $\left(8.5 \times 8.5 \times 7.5 \mathrm{~cm}^{3}\right)$ with standard potting mixture (Klasmann-Deilmann $\mathrm{GmbH}$, Geeste, Germany), irrigated, and fertilized (liquid fertilizer Flory 2 special, 16-9-22) as required. In a cabinet covered with cellophane to allow for the exchange of air and relative humidity $(\mathrm{RH})$, plants were protected from dust, emissions, and fungal infection. After 8 weeks, the adaxial side of the youngest completely unfolded leaf (leaf insertion 1) and leaf insertion 7 of apple seedlings were used for the experiments.

Contact angle measurements (goniometry). Hydrophobicity of surfaces was assessed quantitatively by a drop shape analysis system (Contact Angle System OCA 30-2, Software SCA 202, DataPhysics Instruments GmbH, Filderstadt, Germany) using the sessile drop method. Samples of apple leaves cut from the central area of the leaf lamina were affixed to glass slides with doublesided adhesive tape (TesaFix, Beiersdorf, Hamburg, Germany) to ensure an even surface. The volume of distilled water droplets was $10 \mu \mathrm{l}$. Contact angles were calculated by using the LaplaceYoung-Fitting method. Measurements were made on the adaxial side of two leaf insertions, representing different leaf ages $(n=6)$. For every developmental stage of leaves, three measurements were made resulting in 18 values per leaf insertion. For all artificial surfaces 12 measurements were made.

Hydrophobicity of $V$. inaequalis conidia was measured by placing a 5- $\mu$ l droplet of distilled water on a uniform layer of conidia affixed to a layer of adhesive tape (contact angle $82.0^{\circ} \pm$ 4.7). Contact angle readings were made immediately after the contact of the droplet and were repeated at a 1-min interval until stability of data.

Volume of $\boldsymbol{V}$. inaequalis conidia. The volume of conidia prior and after hydration was calculated according to Aust (2). The length, width, and depth of conidia were measured and the volume was calculated by using the formula $V=4 / 3 \pi a b c$, where $a$ is the half conidia length, $b$ is the half conidia width, and $c$ is the half conidia depth.

Adhesion of conidia. Conidia of $V$. inaequalis were examined for their ability to adhere to apple leaves, glass microscope slides (Marienfeld, Lauda-Königshofen, Germany), and polystyrene (petri dishes, Greiner Bio-One GmbH, Frickenhausen, Germany). Four $5-\mu 1$ droplets were placed onto the surface and incubated in moisture chambers; the concentration of conidia was 4 to $6 \times$ $10^{4} \mathrm{ml}^{-1}$. At 5, 15, 30, 45, 60, 120, 240, 360, and $480 \mathrm{~min}$ after application, respectively, droplets were washed off with $50 \mathrm{ml}$ of tap water and the number of remaining conidia was determined. Water was delivered from a height of $4 \mathrm{~cm}$ onto the surface held at a $45^{\circ}$ angle. To assess the number of conidia initially delivered onto the surface, the average number of four droplets was determined for each surface without washing.

Inhibitor tests. To determine whether active metabolism was necessary for conidial adhesion, conidia were suspended in aqueous solutions of inhibitors and detergents, respectively, and the standard adhesion assay was carried out with a polystyrene surface. Conidia suspensions were prepared with cycloheximide (AppliChem GmbH, Darmstadt, Germany) and chloramphenicol (Fluka $\mathrm{GmbH}$, Buchs, Switzerland) at $10 \mu \mathrm{g} \mathrm{ml}^{-1}$, respectively, actinomycin D at $200 \mu \mathrm{g} \mathrm{ml}^{-1}$ (AppliChem), 5.5\% methanol, 0.2 and $0.02 \%$ sodium dodecyl sulfate (SDS; AppliChem), $0.001 \%$ Tween 80 , and proteinase $\mathrm{K}$ at $10 \mu \mathrm{g} \mathrm{ml}^{-1}$ (AppliChem). All solids were dissolved in distilled water. For all adhesion experiments, 4 droplets were counted at each time interval using a light microscope with $\times 1,000$ magnification.

Light microscopy. Droplets (5 or $20 \mu \mathrm{l}$ ) of conidial suspensions $\left(2.5 \times 10^{4} \mathrm{ml}^{-1}\right.$ of distilled water $)$ were placed on glass slides and polystyrene and incubated in moisture chambers at $18^{\circ} \mathrm{C}$ with $16 \mathrm{~h}$ light. Conidia were stained with a range of dyes specific for biochemical components that might occur in any STM (Table 1): 0.2\% Coomassie brilliant blue R 250 (Fluka), 1\% Alcian blue 8 GS (Fluka) in 5\% acetic acid, $0.01 \%$ fuchsin acid (Merck, Darmstadt, Germany) in lactophenol, $0.1 \%$ Congo red (Serva, Heidelberg, Germany) (0.1 g of Congo red, $0.05 \mathrm{ml}$ of ethanol, and $100 \mathrm{ml}$ of distilled water), $0.01 \%$ GS-II lectin from Griffonia simplicifolia, $0.01 \%$ peanut agglutinin (PNA), $0.01 \%$ Concanavalin A (Con A), or $0.01 \%$ wheat germ agglutinin (WGA). Lectins were supplied by Molecular Probes (Leiden, NL). The samples treated with lectins were observed with the filter combination FI/RH (BP 490-560/FT 500/LP 525-605; Leica, Wetzlar, Germany) and N3 (BP 546/12/FT 565/BP 600/40; Leica), respectively, in the fluorescence mode. Lectins were linked with the following dyes: GS-II and PNA; Alexa Fluor (488 nm) and Con

TABLE 1. Histochemical labeling of the adhesive material released by the tips of conidia of Venturia inaequalis

\begin{tabular}{|c|c|c|}
\hline Dye/lectin & Binding position & $\begin{array}{l}\text { Labeling of } \\
\text { adhesive }\end{array}$ \\
\hline \multicolumn{3}{|l|}{ Dyes } \\
\hline Alcian blue & Mucopolysaccharide, mucin & Positive \\
\hline Congo red & Amyloidic carbohydrates & Positive \\
\hline Coomassie blue & Proteins & Positive \\
\hline Acid fuchsin & Carbohydrates & Negative \\
\hline \multicolumn{3}{|l|}{ Lectins } \\
\hline Concanavalin A & $\begin{array}{c}\alpha \text {-D-Mannose, } \alpha \text {-D-glucose, and } \\
\alpha \text {-D- } N \text {-acetylglucosamine }\end{array}$ & Negative \\
\hline $\begin{array}{l}\text { GS-II from } \\
\text { Griffonia simplicifolia }\end{array}$ & $\begin{array}{l}\text { Terminal, nonreducing } \alpha \text { - or } \\
\beta-N \text {-acetyl-D-glucosaminyl } \\
\text { residues of glycoproteins }\end{array}$ & Positive \\
\hline Peanut agglutinin & $\begin{array}{l}\text { Terminal } \beta \text {-galactose residues } \\
\text { of glycoproteins }\end{array}$ & Positive \\
\hline Wheat germ agglutinin & $\begin{array}{c}\beta \text {-(D-1,4- } N \text {-acetylglucosamin })_{3} \text { and } \\
\beta \text {-(D-1,4- } N \text {-acetylglucosamin })_{2} \\
N \text {-acetylneuramine acid }\end{array}$ & Negative \\
\hline
\end{tabular}


A; Texas red (543 nm) and WGA; and Oregon green (488 nm). Conidia were observed with a Leitz microscope (DMRB Leica) equipped with Nomarski interference contrast and epifluorescence optics. The quality of light micrographs of conidia on glass slides was higher than that of conidia on polystyrene because of the unfavorable refraction index of polystyrene. In this study, we therefore preferred glass surfaces for the documentation of the staining results.

A stereomicroscope (MZ16 F, Leica Microsystems) was used to observe freshly harvested conidia of $V$. inaequalis without sample preparation. The samples were observed using $\times 500$ magnification.

Scanning electron microscopy (SEM). For SEM (JEOL JSM5600 LV, Eching, Germany), conidia were placed on a polycarbonate adhesive tape and sputtered with gold (30 mA for 100 s, Sputter SCD 040, Balzers Union, Liechtenstein). Dry conidia were observed as well as conidia incubated in a droplet of distilled water for $5 \mathrm{~min}$. Immediately before sputtering, the water was soaked up with filter paper. Samples were observed at 7 to $9 \mathrm{kV}$ under high vacuum conditions at ambient temperature.

Transmission electron microscopy (TEM). Dry conidia from infected leaves were brushed on glass slides and incubated in a steam of osmium tetraoxide $\left(\mathrm{OsO}_{4}\right)$ for $24 \mathrm{~h}$ at room temperature for previous fixation. In order to study hydrated conidia, spores were placed on filter paper folded as a small bag and held in water for $5 \mathrm{~s}$ and for $1 \mathrm{~h}$, respectively. Dry and hydrated conidia were embedded in agar (SEA Plaque Agarose, low melting) and placed in a fixing solution of $8 \%$ formaldehyde and $8 \%$ glutaraldehyde in $0.2 \mathrm{M}$ cacodylic acid sodium salt trihydrate buffer ( $\mathrm{pH} 7.3$ ) (18) for $2.5 \mathrm{~h}$ at room temperature. Samples were then rinsed five times in $0.2 \mathrm{M}$ cacodylic acid sodium salt trihydrate buffer ( $\mathrm{pH} 7.3$ ), and further fixed in osmium tetraoxide solution of $0.4 \% \mathrm{KCr}_{2} \mathrm{O}_{7} / 3.4 \% \mathrm{NaCl} / 2 \% \mathrm{OsO}_{4}(1: 1: 2$, vol/vol/vol $)$ according to Dalton (8). The samples were rinsed eight times in cacodylic acid sodium salt trihydrate butter $(\mathrm{pH}$ 7.3) followed by dehydration in increasing concentrations of ethanol/double-distilled water $(15,30,50,70,90$ and $100 \%)$ for 20 min per concentration. The embedding of samples occurred by infiltration in increasing concentrations of Spurr's ERL-resin. The ERL-resin consisted of $10.0 \mathrm{~g}$ of ERL-4206, $4.0 \mathrm{~g}$ of DER 736, and $0.4 \mathrm{~g}$ of S-1 (33). At $4{ }^{\circ} \mathrm{C}$, samples were put in different ratios of ERL-resin/ propylene oxide ( $\mathrm{vol} / \mathrm{vol})$ in the following order: $1: 3$ for $16 \mathrm{~h}$, 1:1 for $8 \mathrm{~h}, 3: 1$ for $16 \mathrm{~h}$. The polymerization in 100\% ERL-resin was carried out in flat embedding trays (Agar Aids Ltd., Stansted, $\mathrm{UK}$ ) at $70^{\circ} \mathrm{C}$ for $8 \mathrm{~h}$. Ultra-thin sections of 62 to $66 \mathrm{~nm}$ thickness were cut with a Reichert-Jung Ultramicrotome Ultracut E (Heidelberg, Germany) with a diamond knife and placed on nickel grids.

Specimens were contrasted according to Geyer (13). The grids were placed in $2 \%$ uranyl acetate for $8 \mathrm{~min}$ and then rinsed in double-distilled water twice. The grids were transferred in a lead acetate solution according to Reynolds (29) for $2 \mathrm{~min}$ and then rinsed twice in double-distilled water. The lead acetate solution consisted of $1.33 \mathrm{~g}$ of $\mathrm{Pb}\left(\mathrm{NO}_{3}\right)_{2}$ and $1.76 \mathrm{~g}$ of $\mathrm{Na}_{3}\left(\mathrm{C}_{6} \mathrm{H}_{5} \mathrm{O}_{7}\right) \times$ $2 \mathrm{H}_{2} \mathrm{O}$ in $30 \mathrm{ml}$ of double-distilled water and was thoroughly mixed and stored in darkness for $30 \mathrm{~min}$. The $\mathrm{pH}$ value of 12 was adjusted using $1 \mathrm{~N} \mathrm{NaOH}$, and the solution was filled with $50 \mathrm{ml}$ of double-distilled water. Grids were allowed to dry and stored at room temperature. Observations were carried out using a Philips CM 10 (Philips, Eindhoven, Netherlands) linked with a Mega View III Soft Imaging System.

Statistics. All experiments were repeated two to three times. All values shown in the figures and in the text represent averages \pm standard error (SE) of the mean. Statistical tests ( $t$ test for pairwise comparisons and Tukey test for multiple comparisons) were conducted using SPSS for Windows, version 12.0 (SPSS Inc., Chicago).

\section{RESULTS}

Hydrophobicity of surfaces. The hydrophobicity of adaxial apple leaf surfaces and model surfaces was assessed goniometrically using the contact angle of water droplets on leaf insertions 1 and 7, glass slides, and polystyrene. With a contact angle of $107.2^{\circ} \pm 5.0$ (mean $\pm \mathrm{SE}$ ), the adaxial surface of the youngest leaf (insertion 1) was significantly more hydrophobic than that of the older leaf insertion $7\left(77.6^{\circ} \pm 13.0\right)$, which showed also higher variability. Hydrophobicity of the model surface polystyrene $\left(88.0^{\circ} \pm 3.3\right)$ was in the same range as the surface of older apple leaves. Glass slides, in contrast, proved to be highly hydrophilic (contact angle $27.2^{\circ} \pm 4.9$ ).

Characterization of $\boldsymbol{V}$. inaequalis conidia. The physical properties of conidia of $V$. inaequalis were characterized before contact with the target surfaces. Conidia freshly brushed off infected apple leaves had a water content of $8.8 \pm 1.9 \%$ (mean \pm SE), irrespective of the RH during sporulation. Conidia were largely dehydrated and shriveled (Fig. 1A). After contact with water the hydration of conidia significantly increased the volume by about $80 \%$ (Table 2). The increase in volume largely resulted from the increase of conidial depth rather than from width and length. Goniometric measurements characterized the dry conidia of $V$. inaequalis as highly hydrophobic. Wetting of the spore surface, however, resulted in a marked increase in the hydrophilic character of the surface; within $10 \mathrm{~min}$ the contact angle decreased from $118.4^{\circ} \pm 25.4$ to $50.4^{\circ} \pm 10.5$.

Characterization of the morphology of dry conidia and the effect of water. Dry conidia harvested by brushing off from sporulating colonies on apple leaves were largely nonturgid. At the conidial apex of $V$. inaequalis, a globular structure, about 1.4 to $1.6 \mu \mathrm{m}$ in diameter, could be identified in this study for the first time (Fig. 1A). The apical vesicle of young conidia still fixed to the conidiophores was less pronounced (Fig. 1B). TEM images revealed an extracellular matrix often divided into two areas differing in electron density, which was not surrounded by a membrane (Fig. 1C). Immediately after contact with liquid water, conidia became turgid and the apical vesicles became undetectable in SEM (Fig. 1D). On the tip of hydrated conidia, no matrix material was detectable using TEM (Fig. 1E).

Images from a high-resolution stereomicroscope provided evidence that the globular structures on conidial tips were not artifacts produced during sample preparation and SEM observations. Dry conidia on a glass surface were shriveled, however, showed the same globular structures on their tips as seen in SEM (Fig. $1 F)$. After contact with liquid water, conidia immediately became turgid and STG disappeared. Prior to the formation of a germ tube, typically at the conidial tip about 3 to $4 \mathrm{~h}$ after hydration, the conidial tip became broader and rounded. The formation of appressoria was initiated only after 6 to $8 \mathrm{~h}$ of incubation at $100 \%$ RH.

Conidial attachment to different surfaces. On the model surfaces polystyrene and glass, the attachment of conidia differed with hydrophobicity of the surface (Fig. 2A). On hydrophilic glass, adhesion of conidia was low (attached conidia less than 10\%) up to $2 \mathrm{~h}$ of incubation. Only after $4 \mathrm{~h}$ of incubation, corresponding to the beginning of germ tube formation, the degree of adhesion increased to about 53\%; after $6 \mathrm{~h}$ and later, the degree of attachment ranged between 80 and $94 \%$. Within the first $4 \mathrm{~h}$ of incubation the percentage of conidia adherent on the hydrophobic polystyrene surface was significantly higher than that on glass (Fig. $2 \mathrm{~A})$. Fifteen minutes after hydration of conidia about $47 \%$ of the conidia were firmly attached to the hydrophobic surface compared with only $1 \%$ on the hydrophilic surface. After $2 \mathrm{~h}$, the adhesion was as high as that on glass only after $4 \mathrm{~h}$. No significant differences could be observed between both surfaces $6 \mathrm{~h}$ after hydration of conidia. On polystyrene, the degree of adhesion reached the maximum value of $89 \%$. 
The adhesion of $V$. inaequalis conidia on the adaxial side of apple leaves representing different leaf stages was similar for both leaf insertions (Fig. 2B). The degree of adhesion on leaves showed similarities with that observed on the hydrophobic surface of polystyrene. On leaves 1 and 7 almost $40 \%$ of applied conidia adhered within $15 \mathrm{~min}$ after hydration. The maximum attachment reached not more than $75 \%$ of applied conidia, about $15 \%$ less than on polystyrene. The transient, however, significant $(P<0.05)$ decline in the percentage of adherent conidia observed on polystyrene after initial adhesion was delayed and less pronounced on the surface of the oldest apple leaves (leaf 7).

Effect of inhibitors on conidial attachment. Several inhibitors were added to conidial suspensions in order to investigate whether adhesion of conidia is affected by inhibitors of fungal metabolism or whether the putative adhesive is a preformed substance. Data on the adhesion of conidia in the presence of eight inhibitors and detergents after $15 \mathrm{~min}$ and $4 \mathrm{~h}$ of incubation is summarized in Figure 3. The protein biosynthesis inhibitors cycloheximide and chloramphenicol did not show an effect on adhesion of conidia. Similar to conidia without additives, about $50 \%$ of conidia adhered to the surface $15 \mathrm{~min}$ after hydration. Actinomycin D reduced adhesion after 15 min of incubation, but this effect could not be confirmed after $4 \mathrm{~h}$. The effect of methanol, which was used to prepare an aqueous solution of actinomycin D, was not significantly different from the actinomycin D preparation indicating that the solvent rather than the inhibitor of DNA
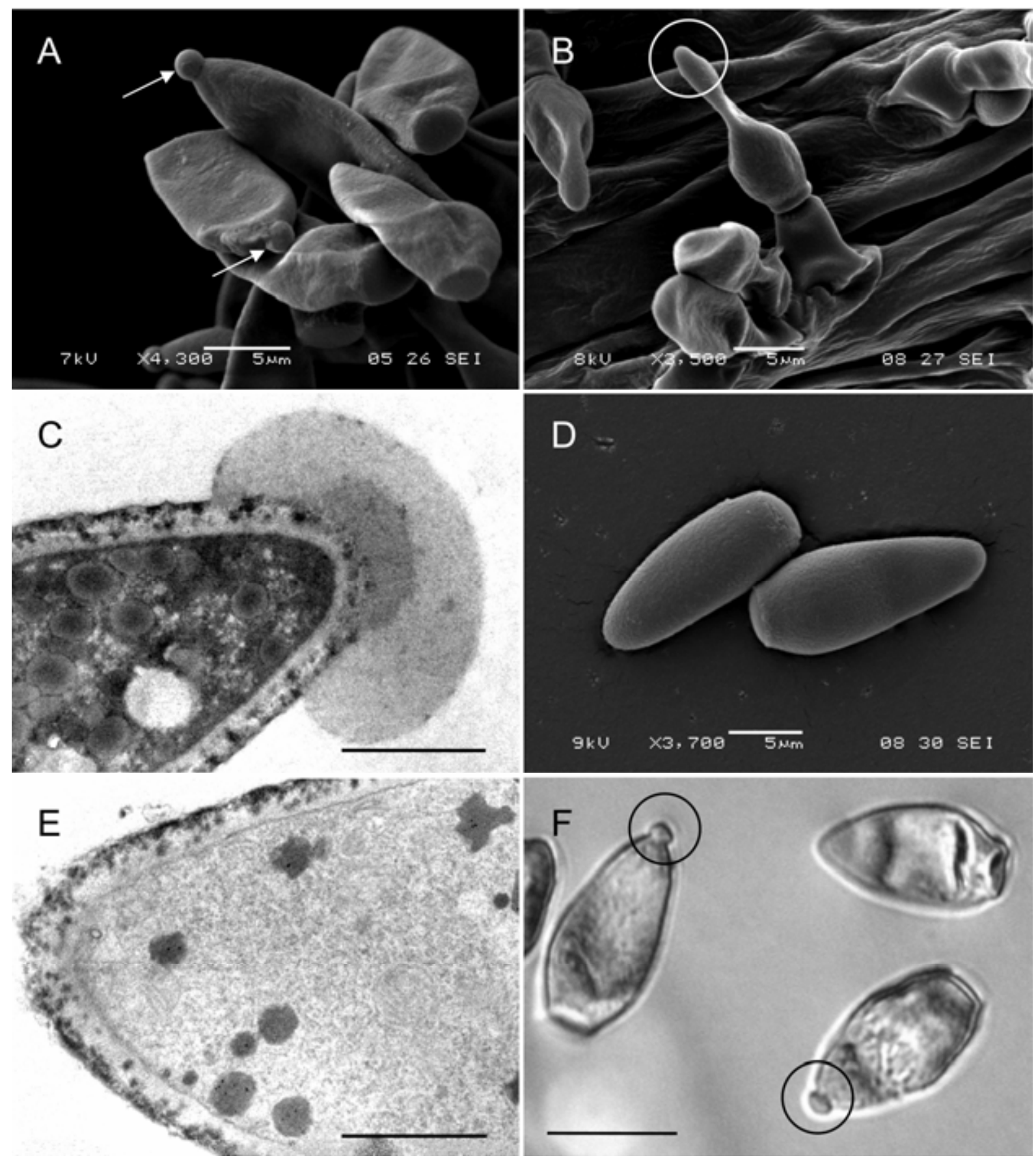

Fig. 1. Morphology of dry and hydrated conidia of Venturia inaequalis. A, The apex of nonturgid conidia consisted of a globular structure (spore tip glue [STG], arrows). B, Nonturgid $V$. inaequalis conidia formed on conidiophores, with small STG (circle). C, Dry conidium showed a two-parted STG (bar represents $1 \mu \mathrm{m}$ ) D, Turgid V. inaequalis conidia after hydration. The vesicles at the spore tip were no longer visible. E, Hydrated conidium without globular structure on the tip (bar represents $1 \mu \mathrm{m}$ ). F, Dry conidia without any preparation showed globular structures (circles) on their apex (bar represents $10 \mu \mathrm{m})(\mathbf{A}, \mathbf{B}$, and $\mathbf{D}$, scanning electron microscopy; $\mathbf{C}$ and $\mathbf{E}$, transmission electron microscopy; and $\mathbf{F}$, light micrograph). 
replication transiently affected conidial adhesion. Only proteinase $\mathrm{K}$ had an effect on the attachment of nongerminated conidia to polystyrene. Also $4 \mathrm{~h}$ after hydration, the percentage of adherent conidia was about $30 \%$ lower than that of conidia without additives.

The addition of the detergent SDS to the suspension also showed a significant effect on conidial adhesion. Conidia were not able to adhere to polystyrene at any time in the presence of $0.2 \%$ SDS (Fig. 3). The use of $0.001 \%$ Tween had an effect similar to SDS.

Site of conidial adhesion. Using light microscopy, the movement of conidia in a stream of water could be observed. As shown in Figure 4, the tip of the conidia adhered to the glass surface whereas the basal part of conidia rotated in the water stream. It seemed that only the tips of conidia were able to adhere to the contact surface. Other light microscopic images demonstrated that conidia of $V$. inaequalis formed aggregates by adhering with the tip of the conidium to the side of another conidium (Fig. 5A). Figure 5 gives evidence for the first time that conidia of $V$. inaequalis release adhesive material for the attachment onto the substratum.

Histochemical characterization of adhesives. Following the washing procedures described above, pads of the putative adhesive material were observed at the tips of almost all adherent conidia. The pads became positively stained for proteins with Coomassie brilliant blue, for amyloidic carbohydrates with Congo red, for mucopolysaccharides with Alcian blue, for $\beta$-galactose residues with PNA, and for terminal $\mathrm{N}$-acetylglucosaminyl residues using GS-II (Fig. 5B to G). Whereas Coomassie blue stained a wide area around the conidial apex (Fig. 5B and C), the Congo red staining was limited to a narrow area at the tip (Fig. 5D). The staining of conidia with Alcian blue was poor and limited to a small area at the tip (Fig. 5E). The lectin PNA showed a stronger staining than GS-II and covered larger parts of the adhesive pad (Fig. 5F). Staining with acid fuchsin and the lectins WGA and Con A gave negative results (Table 1).

\section{DISCUSSION}

For the first time a globular STG on the tapered side of dry $V$. inaequalis conidia was demonstrated that appears similar to STM described for conidia of Magnaporthe grisea (14). After hydration, this adhesive material enables the conidia to adhere to wet hydrophobic surfaces like apple leaves. Dry conidia are more resistant to environmental stress factors than conidia with higher moisture content (15); adhesion and the subsequent germination and penetration of the apple cuticle, processes very sensitive to desiccation, are initiated only after contact with water on the surface.

A sequence of light micrographs of $V$. inaequalis conidia in a stream of water showed adherent conidia, which only rotated with the basal part whereas the tip was fixed at a point to the surface. As Hamer et al. (14) suggested for adhesion of $M$. grisea conidia we also assume for $V$. inaequalis that the ovate to lanceolate shape of conidia as well as the attachment restricted to the apical side of conidia only are favorable to minimize their resistance to water flows, which may dislodge other spores from the surface when hit at their broad side.

TABLE 2. Size and volume of conidia of Venturia inaequalis before and after hydration $(n=30$, mean \pm SE, values within a line with the same letter are not significantly different according to Tukey test, $P \leq 0.01$ )

\begin{tabular}{lccc}
\hline Parameter & Before hydration & Hydrated for 1 min & Hydrated for 6 min \\
\hline Length $(\mu \mathrm{m})$ & $17.7 \pm 0.3 \mathrm{~b}$ & $20.3 \pm 0.4 \mathrm{a}$ & $20.8 \pm 0.4 \mathrm{a}$ \\
Width $(\mu \mathrm{m})$ & $7.9 \pm 0.2 \mathrm{a}$ & $8.3 \pm 0.2 \mathrm{a}$ & $7.8 \pm 0.2 \mathrm{a}$ \\
Depth $(\mu \mathrm{m})$ & $1.9 \pm 0.1 \mathrm{~b}$ & $8.3 \pm 0.2 \mathrm{a}$ & $7.8 \pm 0.2 \mathrm{a}$ \\
Volume $^{\mathrm{z}}\left(\mu \mathrm{m}^{3}\right)$ & $136.8 \pm 5.6 \mathrm{~b}$ & $724.9 \pm 24.9 \mathrm{a}$ & $673.4 \pm 30.1 \mathrm{a}$ \\
\hline
\end{tabular}

z Calculated according to Aust (2).
STG surrounding the conidial tip was detected by several staining techniques on hydrophobic and hydrophilic surfaces and was similar in size and composition on both types of surface. Although the exact chemical composition of the preformed glue is not clear, more than one chemical class seem to be present in the globule and are involved in the gluing process. They are separated from each other, at least before hydration of the spore as indicated in TEM and histochemical experiments. Labeling with PNA and GS-II indicated the presence of glycoproteins in the adhesive material of $V$. inaequalis conidia. Braun and Howard (5) reported proteins and acid mucopolysaccharides to be components of the adhesive matrix of Cochliobolus heterostrophus conidia. Glycoproteins in adhesive material have been described for other plant pathogenic fungi like $N$. haematococca (21), Colletotrichum graminicola (25), and M. grisea (40). The matrix surrounding pycnidiospores of Phyllosticta ampelicida consists partly of acidic polysaccharides as demonstrated by the labeling with several positively charged probes like Alcian blue (20). STG of V. inaequalis could not be labeled by Con A. In contrast, Con A bound heavily to the STM of $M$. grisea conidia indicating the presence of glucose and/or mannose residues, and the presence of Con A resulted in reduced attachment to a hydrophobic surface $(3,14)$. Positive staining of a small area around the apex of conidia with Congo red indicates to amyloidic polysaccharides, however, staining may be also attributed to the amyloid fibrils of polymerized hydrophobins when used in combination with polarized light (24).

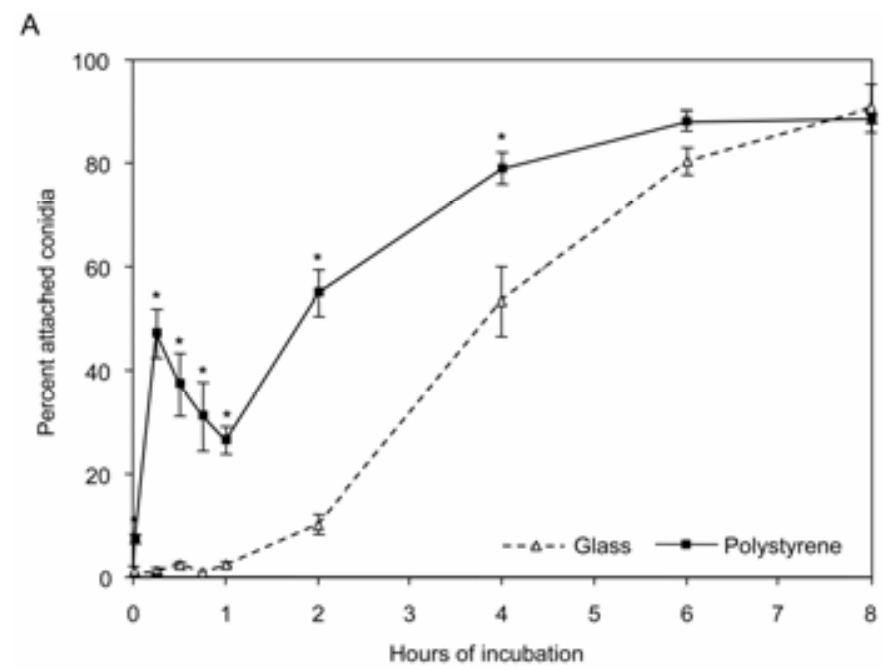

B

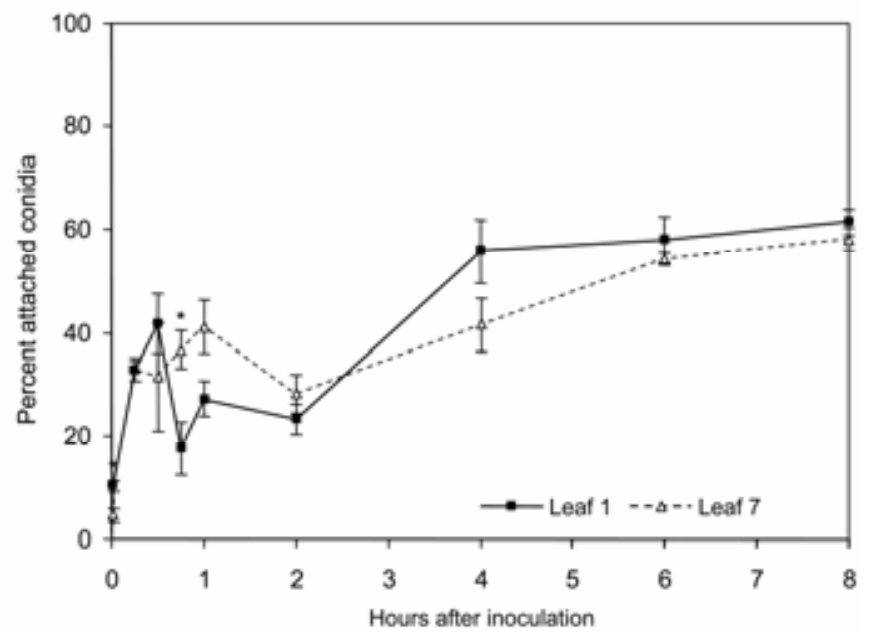

Fig. 2. Effect of surface hydrophobicity (A) and leaf insertion (B) on the attachment of Venturia inaequalis conidia to artificial surfaces and apple leaves, respectively $(n=4$, mean \pm standard error, asterisk at each time indicates significant differences according to $t$ test, $P \leq 0.05$ ). 
Hydrophobins are a family of small, moderately hydrophobic fungal proteins involved in surface interactions $(37,38)$. Class II hydrophobins are able to self-assemble into an amphipathic protein film when confronted with a hydrophilic-hydrophobic interface (37). Rodlet layers of hydrophobins described for conidial surfaces of M. grisea (35) and Aspergillus fumigatus (28) have not been reported for $V$. inaequalis. As nongerminated conidia only adhered to hydrophobic surfaces, not to the hydrophilic glass, it seems unlikely that hydophobins are involved in the primary attachment of $V$. inaequalis.

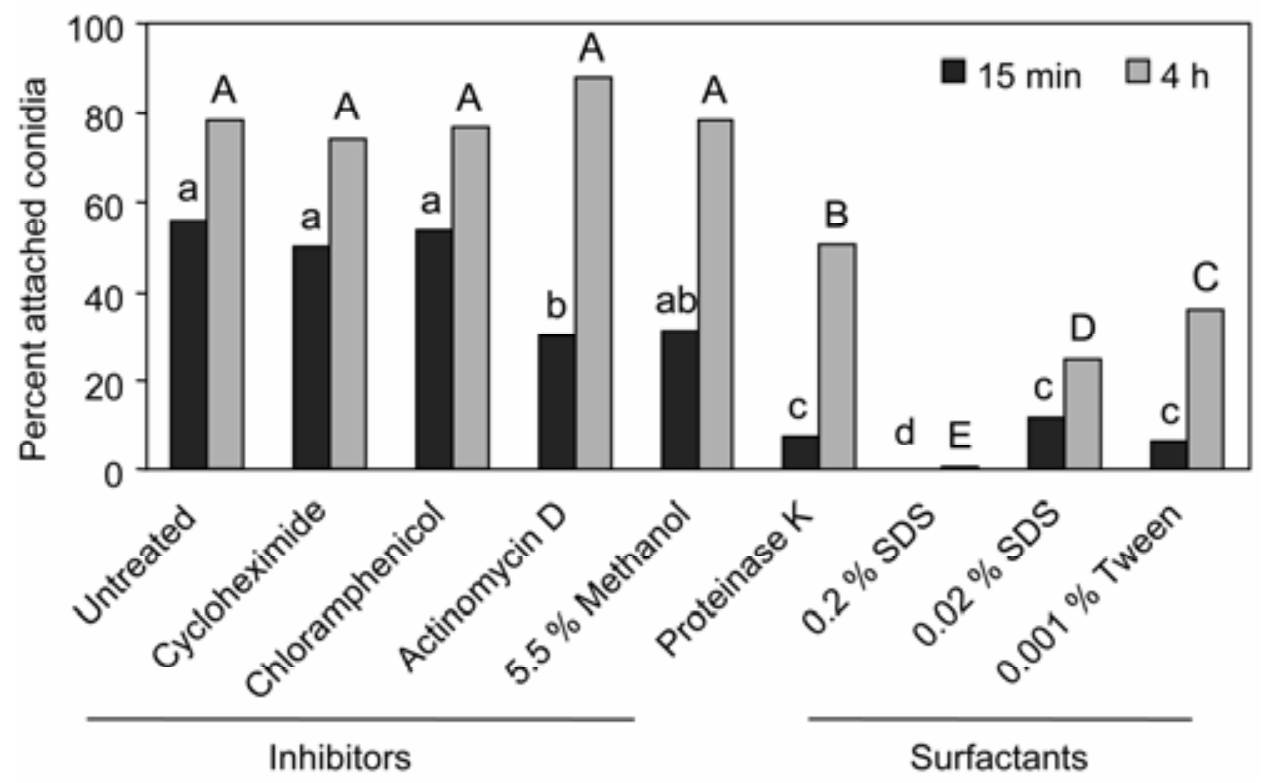

Fig. 3. Effect of inhibitors and surfactants on the attachment of Venturia inaequalis conidia to polystyrene. Droplets of conidia suspension treated with inhibitors were washed off with water $15 \mathrm{~min}$ and $4 \mathrm{~h}$, respectively, after application onto the surface $(n=4$, columns with the same letter are not significantly different according to Tukey test, $P \leq 0.05$ ).
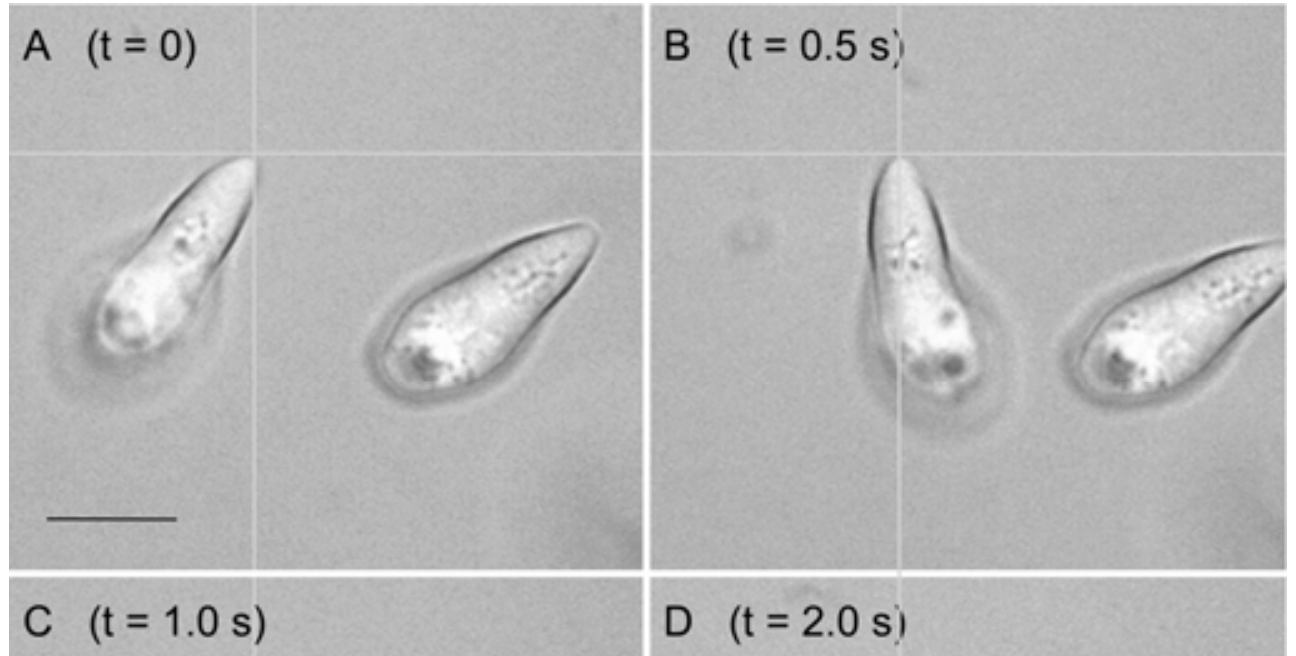

D $(t=2.0 \mathrm{~s})$
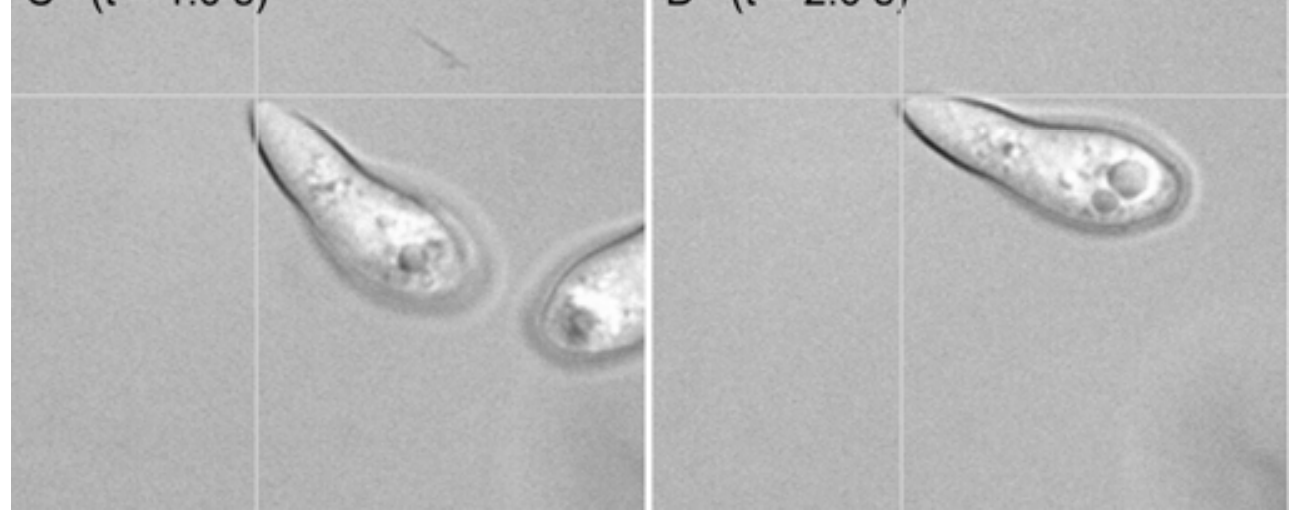

Fig. 4. A to D, Time series on the rotation of an adherent conidium of Venturia inaequalis in a stream of water on a glass surface. Only the tip of the left conidium adhered to the contact surface, allowing the base to rotate freely around the point of fixation until reaching a position providing a minimum flow resistance. The nonadherent conidium on the right was dislodged by the water streaming from the left to the right (light micrographs, bar represents $10 \mu \mathrm{m}$ ). 

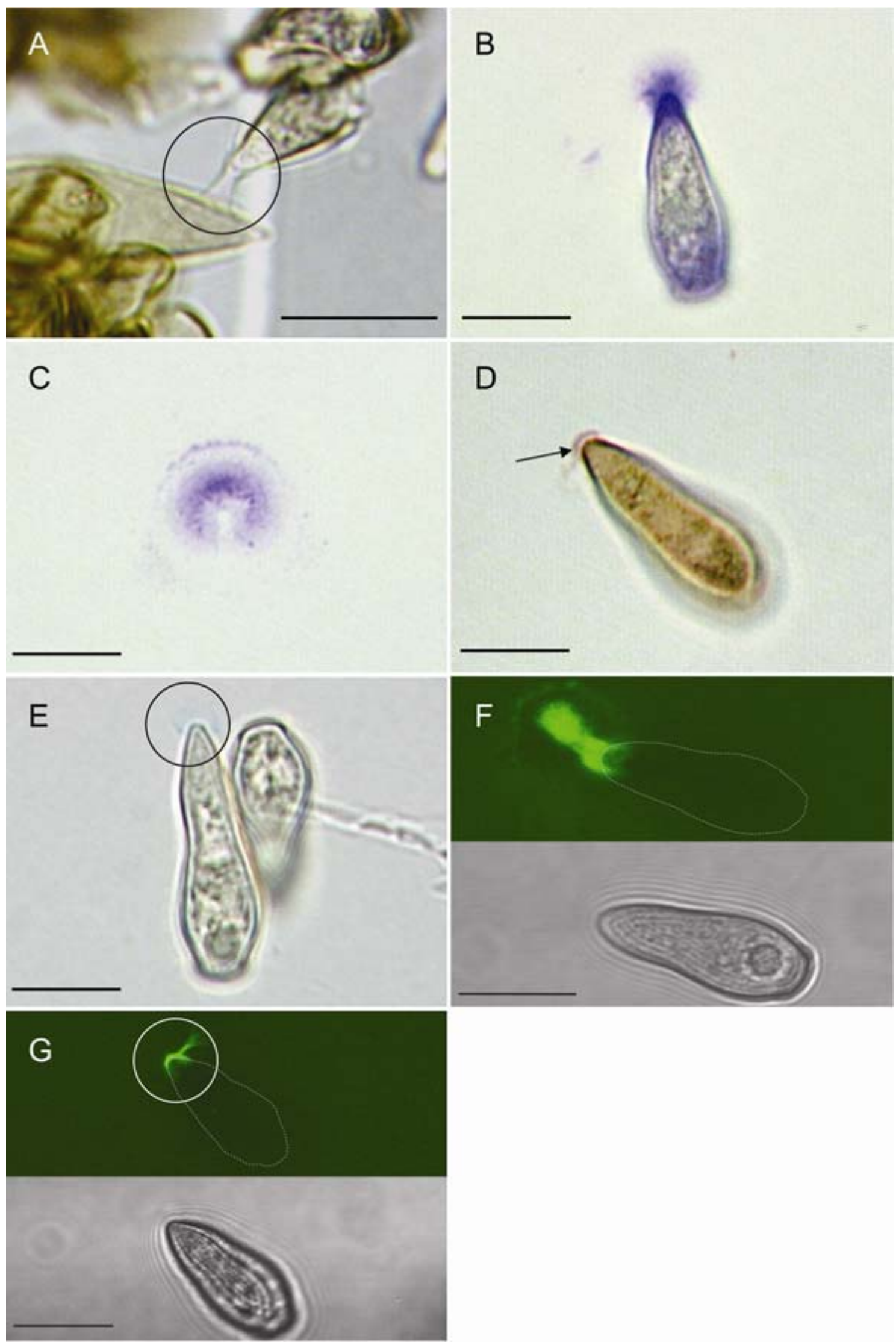

Fig. 5. Conidia of Venturia inaequalis treated with dyes and lectins after adhesion on $\mathbf{A}, \mathbf{B}$, and $\mathbf{D}$, polystyrene and $\mathbf{C}, \mathbf{E}, \mathbf{F}$, and $\mathbf{G}$, glass surface 15 min after application (light micrographs). A, Conidium adherent to another conidium with adhesive material at the conidial tip. B, The adhesive pad at the conidial tip stained with Coomassie brilliant blue. C, Coomassie-stained adhesive pad after removal of conidium. D, With Congo red, only a narrow area at the conidial tip was stainable (arrow). E, Conidia treated with Alcian blue showed an adhesive pad at their tips (circle). F, With peanut agglutinin, a large area of the adhesive pad became visible. G, GS-II was suitable to show the interface between conidial tip and adhesive pad (circle). Bars represent $10 \mu \mathrm{m}$. F and G split images with fluorescence (filter combination FI/RH [top] and brightfield [bottom], contour of conidia for orientation). 
Adhesion of $V$. inaequalis conidia was not influenced by inhibitors like cycloheximide, chloramphenicol, and actinomycin $\mathrm{D}$, respectively. Hence, conidial STG of $V$. inaequalis is preformed and must not be produced after contact to a surface as described for Cochliobolus heterostrophus (5). This is similar to M. grisea for which STM release and spore attachment occur independent of fungal metabolism. Inhibitors of fungal respiration and protein biosynthesis did not disturb the attachment of $M$. grisea conidia (4). In contrast, inhibitors of glycoprotein transport and cycloheximide reduced conidial adhesion of Colletotrichum graminicola by 30 and $50 \%$, respectively, whereas sodium azide had no effect on adhesion (26). Addition of surfactants like SDS and Tween to conidial suspensions significantly decreased the adhesion of nongerminated $V$. inaequalis conidia. Similarly, adhesion of nongerminated conidia of Colletotrichum musae to a hydrophobic surface was reduced by the addition of SDS to the suspension, without affecting germination (30).

STG of $V$. inaequalis seemed to be surrounded neither by a membrane nor by the cell wall of the conidium. In contrast, $M$. grisea releases STM which is produced in the periplasmatic space within conidia through rupture of the outer spore wall of hydrated spores (14). Hence, STG of $V$. inaequalis seems to be a matter of glue droplet outside the conidium, which is still stable in water and not released until contact to a solid surface. The formation of STG should be associated with the secretion of glue material from the spore's cytoplasm through pores of the cell wall.

Hydration of dry conidia was immediately after contact with liquid water (100\% RH was not effective) and was associated with the release of the adhesive material onto an underlying surface. Conidia, which had been shaken in a suspension for some hours, subsequently were able to attach firmly to hydrophobic surfaces indicating that STG was still intact in the suspension (data not shown). Not only hydration but also a contact surface is necessary for STG release as the first step in the host pathogen interaction. The adhesive strength of the glue to a surface depends on its hydrophobicity. Similar to $V$. inaequalis, adhesion of conidia from various phytopathogenic fungi was stronger on hydrophobic surfaces than on hydrophilic surfaces $(1,10,14,26,30,31)$. In general, the cuticle of plant surfaces is hydrophobic and this barrier against water loss (from inside the leaf) and pathogen attack (from the environment) has to be recognized and used by potential pathogens as the first substrate.

In contrast, on hydrophilic surfaces the adhesion of conidia was only minor although the adhesive material was released onto the surface similar to that on hydrophobic surfaces. Initial attachment of $V$. inaequalis to wet host surfaces results from the coincidence of preformed glue (of the pathogen), the hydrophobic cuticle (of the host plant), and the availability of water. The success of adhesion seems to be random and is determined by the plant's surface properties and does not require active recognition of the host plant. Active production and release of ECM after recognition of a suitable surface as described for $C$. graminicola and $N$. haematococca $(17,26)$ is not involved.

Hardness of the surface described to be crucial for the attachment of spores, initiation of spore germination in $C$. graminicola (6), and for appressorium differentiation in M. grisea (41) seems to be of minor importance for adhesion of $V$. inaequalis conidia. Glass is harder than polystyrene and apple leaves; however, adhesion was better on hydrophobic surfaces than on glass. During germ tube formation, however, infection structures of $V$. inaequalis were tightly attached also to the hydrophilic surface, probably due to the secretion of an extracellular matrix by the germ tubes. On apple cuticles (and polystyrene), the protein-containing matrix seems to be largely limited to the tip of the germ tube. Adhesion to the surface was not required for successful germination of conidia as demonstrated by the high germination rate on glass and other surfaces like agar (34). Similarly, adhesion of conidia of B. cinerea and B. sorokiniana has been described as a two-step process; primary attachment of nongerminated conidia upon hydration followed by the formation of ECM by germinating conidia. For both fungi, initial attachment was weak $(1,10)$. As ECM formation by $V$. inaequalis was not influenced by surface hydrophobicity, the adhesion of fungal structures in later fungal growth stages was very similar on all surfaces.

The transient, however, reproducible decline in the adhesion of $V$. inaequalis conidia after about 1 to $2 \mathrm{~h}$ of incubation was more pronounced on highly hydrophobic surfaces (young leaves, polystyrene) than on older, less hydrophobic leaves and was absent on glass. It coincided with a very early stage of germination characterized by the rounding of the conidial tip. During swelling of the germination pore and beginning of germination, the strength of adhesion due to STG spatially limited to the conical tip of conidia seems to be transiently weakened. This impairment may result from the fact that both processes, adhesion and germination, take place at the same site of conidia.

$V$. inaequalis has evolved a mechanism for the attachment of nongerminated conidia primarily to hydrophobic surfaces. Conidia are provided with a preformed droplet of STG that successfully interacts with a solid substratum only in the presence of water required for the subsequent formation of germ tubes.

\section{ACKNOWLEDGMENTS}

The support of R. Pontzen, Bayer CropScience, Monheim, Germany, in enabling the SEM investigations is gratefully acknowledged. We thank J. Hamacher (Institute of Crop Science and Resource Conservation, Phytomedicine, University of Bonn) and J. Bedorf (Institute of Pathology, University-Hospital Bonn) for the support in transmission electron microscopy, and I. Neukirchen (INRES - Phytomedicine) for technical assistance. Financial support of C. Schumacher by the University of Bonn is gratefully acknowledged.

\section{LITERATURE CITED}

1. Apoga, D., Jansson, H. B., and Tunlid, A. 2001. Adhesion of conidia and germlings of the plant pathogenic fungus Bipolaris sorokiniana to solid surfaces. Mycol. Res. 105:1251-1260.

2. Aust, H. J. 1981. Über den Verlauf von Mehltauepidemien innerhalb des Agro-Ökosystems Gerstenfeld. Acta Phytomed. Heft 7, Verlag Paul Parey, Berlin.

3. Bourett, T. M., Picollelli, M. A., and Howard, R. J. 1993. Postembedment labeling of intracellular Concanavalin A-binding sites in freezesubstituted fungal cells. Exp. Mycol. 17:223-235.

4. Braun, E. J., and Howard, R. J. 1994. Adhesion of fungal spores and germlings to host-plant surfaces. Protoplasma 181:202-212.

5. Braun, E. J., and Howard, R. J. 1994. Adhesion of Cochliobolus heterostrophus conidia and germlings to leaves and artificial surfaces. Exp. Mycol. 18:211-220.

6. Chaky, J., Anderson, K., Moss, M., and Vaillancourt, L. 2001. Surface hydrophobicity and surface rigidity induce spore germination in Colletotrichum graminicola. Phytopathology 91:558-564.

7. Cotter, G., and Kavanagh, K. 2000. Adherence mechanisms of Candida albicans. Brit. J. Biomed. Sci. 57:241-249.

8. Dalton, A. J. 1955. A chrome-osmium fixative for electron microscopy. Anat. Rec. 121: 281

9. Deising, H., Nicholson, R. L., Haug, M., Howard, R. J., and Mendgen, K. 1992. Adhesion pad formation and the involvement of cutinase and esterases in the attachment of uredospores to the host cuticle. Plant Cell 4:1101-1111.

10. Doss, R. P., Potter, S. W., Chastagner, G. A., and Christian, J. K. 1993. Adhesion of nongerminated Botrytis cinerea conidia to several substrata. Appl. Environ. Microbiol. 59:1786-1791.

11. Epstein, L., and Nicholson, R. L. 1997. Adhesion of spores and hyphae to plant surfaces. Pages 11-25 in: The Mycota V: Plant Relationships, Part A. G. Caroll and P. Tudzynski, eds. Springer, Berlin, Germany.

12. Evans, R. C., Stempen, H., and Frasca, P. 1982. Evidence for a twolayered sheath on germ tubes of three species of Bipolaris. Phytopathology 72:804-807.

13. Geyer, G. 1973. Ultrahistochemie. Gustav Fischer, Stuttgart.

14. Hamer, J. E., Howard, R. J., Chumley, F. G., and Valent, B. 1988. A mechanism for surface attachment in spores of a plant pathogenic fungus. Science 239:288-290. 
15. Hong, T. D., Ellis, R. H., and Moore, D. 1997. Development of a model to predict the effect of temperature and moisture on fungal spore longevity. Ann. Bot. 79:121-128.

16. Huynh, M. H., Rabenaus, K. E., Harper, J. M., Beatty, W. L., Sibley, L. D., and Carruthers, V. B. 2003. Rapid invasion of host cells by Toxoplasma requires secretion of the MIC2-M2AP adhesive protein complex. EMBO J. 22:2082-2090.

17. Jones, M. J., and Epstein, L. 1989. Adhesion of Nectria haematococca macroconidia. Physiol. Mol. Plant Pathol. 35:354-461.

18. Karnovsky, M. J. 1965. A formaldehyde glutaraldehyde fixative of high osmolality for use in electron microscopy. J. Cell. Biol. 27:137-138.

19. Klein, B. S. 2000. Molecular basis of pathogenicity in Blastomyces dermatitidis: The importance of adhesion. Curr. Opin. Microbiol. 3:339343.

20. Kuo, K. C., and Hoch, H. C. 1995. Visualization of the extracellular matrix surrounding pycnidiospores, germlings, and appressoria of Phyllosticta ampelicida. Mycologia 87:759-771.

21. Kwon, Y. H., and Epstein, L. A. 1993. 90-kDa glycoprotein associated with adhesion of Nectria haematococca macro-conidia to substrata. Mol. Plant-Microbe Interact. 6:481-487.

22. MacHardy, W. E. 1995. Apple Scab: Biology, Epidemiology, and Management. American Phytopathological Society, St. Paul, MN.

23. MacHardy, W. E., and Gadoury, D. M. 1989. A revision of Mills's criteria for predicting apple scab infection periods. Phytopathology 79:304-310.

24. Makay, J. P., Matthews, J. M., Winefield, R. D., Makay, L. G., Haverkamp, R. G., and Templeton, M. D. 2001. The hydrophobin EAS is largely unstructured in solution and functions by forming amyloid-like structures. Structure 9:83-91.

25. Mercure, E. W., Kunoh, H., and Nicholson, R. L. 1995. Visualization of materials released from adhered, ungerminated conidia of Colletotrichum graminicola. Physiol. Mol. Plant Pathol. 46:121-135.

26. Mercure, E. W., Leite, B., and Nicholson, R. L. 1994. Adhesion of ungerminated conidia of Colletotrichum graminicola to artificial hydrophobic surfaces. Physiol. Mol. Plant Pathol. 45:421-440.

27. Nicholson, R. L., and Epstein, L. 1991. Adhesion of fungi to the plant surface. Pages 3-23 in: The Fungal Spore and Disease Initiation in Plants and Animals. G. T. Cole and H. C. Hoch, eds. Plenum Press, New York.

28. Paris, S., Debeaupuis, J.-P., Crameri, R., Carey, M., Charlès, F., Prévost, M. C., Schmitt, C., Philippe, B., and Latgé, J. P. 2003. Conidial hydrophobins of Aspergillus fumigatus. Appl. Environ. Microbiol. 69:15811588
29. Reynolds, E. S. 1963. The use of lead citrate at high $\mathrm{pH}$ as an electron opaque stain in electron microscopy. J. Cell. Biol. 17:208-21.

30. Sela-Buurlage, M. B., Epstein, L., and Rodriguez, R. J. 1991. Adhesion of ungerminated Colletotrichum musae conidia. Physiol. Mol. Plant Pathol. 39:345-352.

31. Shaw, B. D., Carroll, G. C., and Hoch, H. C. 2006. Generality of the prerequisite of conidium attachment to a hydrophobic substratum as a signal for germination among Phyllosticta species. Mycologia 98:186194.

32. Shaw, B. D., and Hoch, H. C. 2003. Ions as regulators of growth and development. Pages 73-89 in: The Mycota VII: Biology of the Fungal Cell. R. J. Howard and N. A. R. Gow, eds. Springer, Heidelberg New York.

33. Spurr, A. R. 1969. A low viscosity epoxy resin embedding medium for electron microscopy. J. Ultrastruct. Res. 26:31-43.

34. Steiner, U., and Oerke, E.-C. 2007. Localized melanization of appressoria is required for pathogenicity of Venturia inaequalis. Phytopathology 97:1222-1230.

35. Talbot, N. J., Kershaw, M. J., Wakley, G. E., de Vries, O. M. H., Wessels, J. G. H., and Hamer, J. E. 1996. MPG1 encodes a fungal hydrophobin involved in surface interactions during infection-related development of Magnaporthe grisea. Plant Cell 8:985-999.

36. Tucker, S. L., and Talbot, N. J. 2001. Surface attachment and prepenetration stage development by plant pathogenic fungi. Annu. Rev. Phytopathol. 39:385-417.

37. Wessels, J. G. H. 1997. Hydrophobins: Proteins that change the nature of the fungal surface. Adv. Microbiol. Physiol. 38:1-45.

38. Wösten, H. A. B., Schuren, F. H. J., and Wessels, J. G. H. 1994. Interfacial self-assembly of a hydrophobin into an amphipathic protein membrane mediates fungal attachment to hydrophobic surfaces. EMBO J. 13:58485854.

39. Wright, A. J., Thomas, B. J., and Carver, T. L. W. 2002. Early adhesion of Blumeria graminis to plant and artificial surfaces demonstrated by centrifugation. Physiol. Mol. Plant Pathol. 61:217-226.

40. Xiao, J. Z., Ohshima, A., Kamakura, T., Ishiyama, T., and Yamaguchi, I. 1994. Extracellular glycoprotein(s) associated with cellular differentiation in Magnaporthe grisea. Mol. Plant-Microbe Interact. 7:639-644.

41. Xiao, J. Z., Watanabe, T., Kamakura, T., Ohshima, A., and Yamaguchi, I. 1994. Studies on cellular differentiation of Magnaporthe griseaPhysicochemical aspects of substratum surfaces in relation to appressorium formation. Physiol. Mol. Plant Pathol. 44:227-236. 